\title{
Structural Characterization of the Homeodomain Transcription Factor Gsx2 Bound to DNA Jordan Webb ${ }^{1}$, Ellie Kolb², Rhett Kovall ${ }^{3}$ \\ ${ }^{1}$ University of Cincinnati ${ }^{2}$ University of Cincinnati, ${ }^{3}$ University of Cincinnati webbja96@gmail.com
}

Gsx2 is a conserved homeodomain transcription factor required for the patterning and differentiation of neural progenitors within the lateral ganglionic eminence of the ventral telencephalon, and subsequent development of the basal ganglia. This role of Gsx2 is highlighted by two patients harboring different Gsx2 variants, both resulting in nearly complete basal ganglia agenesis, thus causing severe intellectual disabilities and dystonia. One patient is homozygous for a GSX2 variant that generates a premature stop-codon after the 9th amino acid, effectively resulting in a GSX2 null allele, while the other patient is homozygous for a single missense variant: GSX2 Q251R. Our preliminary studies suggest that altered DNA binding specificity of this missense variant may be the primary driver of the underdeveloped basal ganglia phenotype. In addition, we previously found that Gsx2 binds DNA via two distinct mechanisms: as a monomer to independent AT-rich sites (herein called monomer (M) sites) and as cooperative homodimers to two precisely spaced and oriented AT-rich sites (herein called dimer (D) sites). Importantly, Gsx2 mediates opposing transcriptional outcomes via each type of site: repression via M-sites vs gene stimulation via D-sites. Moving forward, we aim to investigate the molecular mechanisms, including both specificity and cooperativity, underlying Gsx2 function through rigorous characterization of wildtype Gsx2 and these patientderived variants using structural biology and biophysical approaches. To this end, we have solved the first structure, to our knowledge, of the homeodomain of Gsx2 bound to DNA using X-ray crystallographic techniques. This structure may not answer all of our many questions, however, does provide several key points of interest, as well as being a stepping-stone towards elucidating and characterizing additional structures of wildtype and mutant Gsx 2 in both monomer and dimer configurations on DNA.

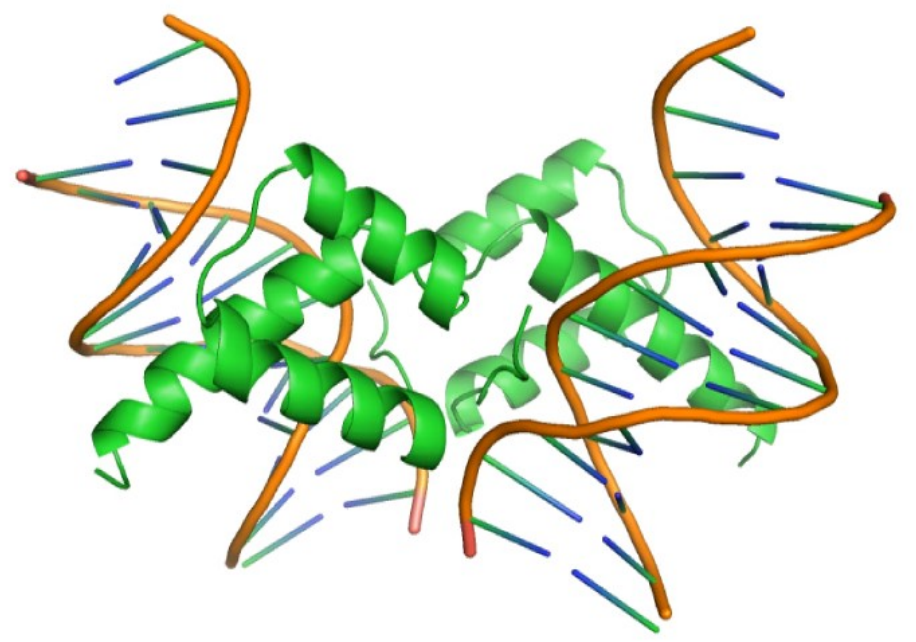

Figure 1 